\title{
De los grafemas a la era digital. Revista Colombiana de Gastroenterología: 35 años
}

\section{From graphs to the digital age. Revista Colombiana de Gastroenterología: 35 years}

Robin Germán Prieto-Ortiz. ${ }^{*}$

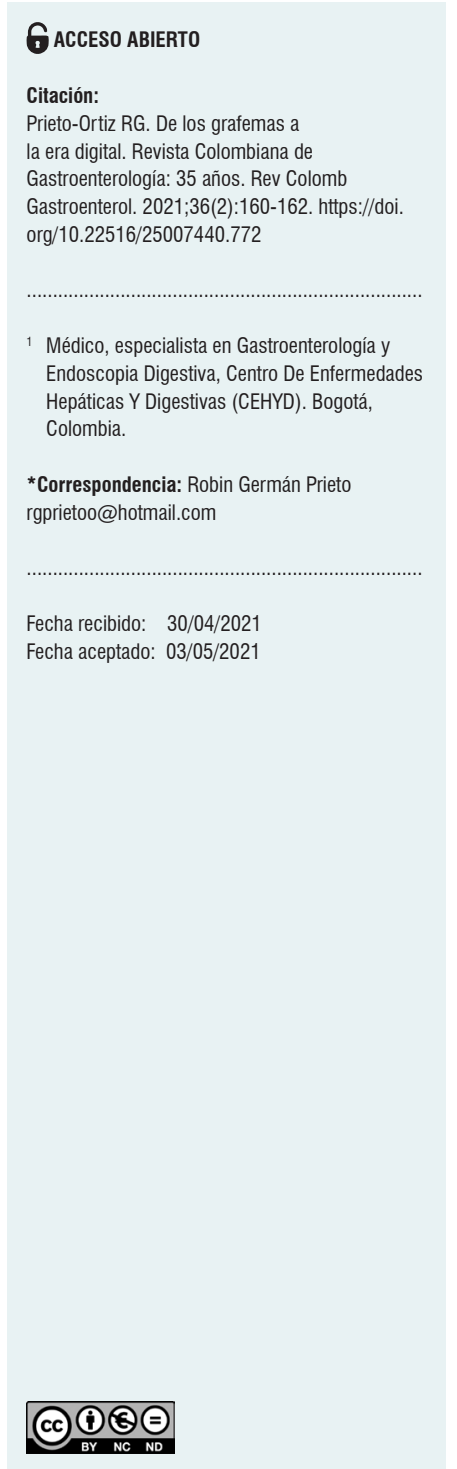

La frase "Las palabras vuelan, lo escrito permanece" es atribuida a Cayo Tito, quien las pronunció hace más de 20 siglos ante el Senado Romano. Hasta nuestros tiempos ha llegado como "lo escrito, escrito está" o "a las palabras se las lleva el viento".

No es tan simple pensar que primero fue el lenguaje hablado y posteriormente el lenguaje escrito. De hecho, algunos semiólogos del siglo XX pensaban que primero fue la escritura antes que el lenguaje verbal, pero actualmente, junto con los lingüistas, consideran probado que la escritura es posterior al lenguaje verbal articulado. Se cree que el origen de la escritura conformada por logogramas y grafemas se remonta al siglo IV A. C. y que apareció de forma aislada pero simultánea en China, Egipto (logogramas y grafemas) y Mesopotamia (grafemas). En América apareció posteriormente mediante la escritura jeroglífica de los Mayas.

A pesar de la invención de la imprenta en el siglo XV y aunque inicialmente existieron muchos textos médicos, la comunicación de los adelantos o invenciones científicas continuó mediante la escritura de cartas y de textos escritos a mano o manuscritos, y solo hasta el siglo XVII las sociedades científicas crearon las primeras revistas. Le Journal des Sçavans, en París, y Philosophical Transactions of the Royal Society, en Londres (1665) y Acta Eruditorum Lipsiensis, en Leipzig (1682) (1).

Las revistas médicas propiamente dichas se fundaron a finales del siglo XVIII en Europa y posteriormente en Estados Unidos y Canadá. De las revistas fundadas en el siglo XVIII solo 24 siguen activas; de esas, las más familiares para nosotros son The New England Journal of Medicine (1812), de Boston; The Lancet (1823), de Londres; y British Medical Journal (1840), de Londres. A mediados del siglo XX, las revistas médicas adoptaron el método experimental para la investigación científica y el formato de resumen en inglés o abstract, introducción, materiales y métodos, resultados y discusión, seguidos por las referencias; y se empezó a realizar la evaluación de los revisores pares, lo que permitió la selección y favoreció la calidad de los artículos publicados (2).

En la última década del siglo XX comenzaron a aparecer las revistas electrónicas como publicaciones simultáneas a la versión impresa, o como única forma de publicación. Lo que, junto con el uso de canales virtuales y redes sociales en los últimos años, ha favorecido la difusión del contenido de las revistas y, por tanto, del conocimiento médico.

La Asociación Colombiana de Gastroenterología fue fundada como Sociedad Colombiana de Gastroenterología (SCG) en 1947 y, siguiendo la tradición de las sociedades científicas, promovió la creación de la Revista Colombiana de Gastroenterología, cuyo primer número fue publicado en 1985 (Figura 1), y sus fundadores y dos primeros 
editores fueron los doctores Germán Liévano Rodríguez (vicepresidente de la SCG) y Paulo Emilio Archila Falla (3). Hasta la fecha y luego de 35 años ininterrumpidos, son 13 los editores que han orientado los diversos equipos de trabajo (Tabla 1) y como líderes han favorecido el progreso y avance de la revista, para posicionarla en el lugar que actualmente ocupa. Desde su fundación, la revista ha sido el órgano oficial de las asociaciones de Gastroenterología, Endoscopia y Coloproctología, y desde 1987, de la de Hepatología.

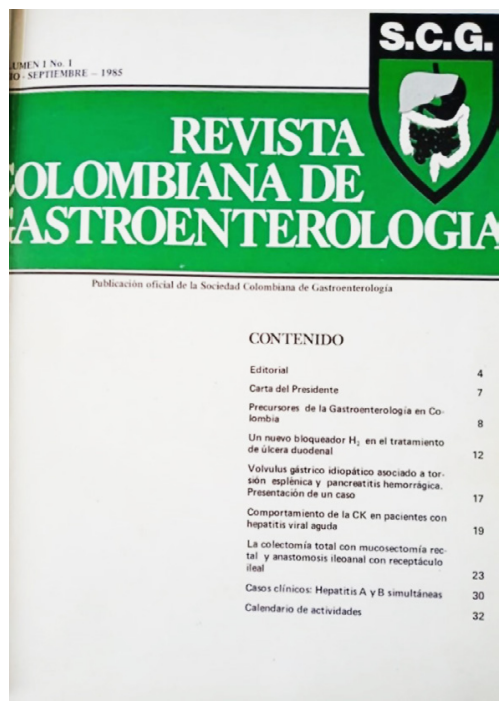

Figura 1. Primer número de la Revista Colombiana de Gastroenterología (julio-septiembre, 1985).

Desde hace poco más de 1 año, bajo la dirección editorial del doctor Diego Aponte, la revista ha tenido una serie de cambios que han permitido su crecimiento y modernización. Uno de los puntos más importantes ha sido el trabajo desarrollado pensando en invertir la pirámide en la que tradicionalmente el ápice es ocupado por el editor y grupo editorial, y la base está conformada por los lectores y autores. El concepto manejado actualmente en la revista es que la punta de la pirámide está ocupada por los lectores y autores, la razón de ser de una revista, en tanto que la base está conformada por el editor y el grupo editorial, quienes, junto con los revisores pares, trabajan pensando en los primeros.

Otro aspecto muy importante en el que se ha venido trabajando es en la academización de la revista, basada en el estudio permanente realizado al interior del equipo, con el fin de comprender el proceso editorial y todo lo que el mismo conlleva. Se ha ampliado el número de revisores pares, quienes entienden que el apoyo que brindan, además de ser todo un honor, agiliza el proceso del que forman parte imprescindible. Apoyados en las redes sociales, se han establecido canales de comunicación más directos y se han realizado reuniones virtuales enfocadas en atraer y fidelizar a nuestros autores y lectores, garantizándoles procesos más dinámicos, ajustados a sus expectativas y a las recomendaciones editoriales internacionales.

Tabla 1. Editores de la Revista Colombiana de Gastroenterología en orden cronológico

\begin{tabular}{|c|c|}
\hline Editor & Período \\
\hline Dr. Germán Liévano Rodríguez & $\begin{array}{l}\text { Julio de } 1985 \text { a diciembre } \\
\text { de } 1985\end{array}$ \\
\hline Dr. Paulo Emilio Archila Falla & $\begin{array}{l}\text { Enero de } 1986 \text { a marzo de } \\
1995\end{array}$ \\
\hline Dr. Carlos Manuel Serrano Mora & $\begin{array}{l}\text { Abril de } 1995 \text { a marzo de } \\
1998\end{array}$ \\
\hline Dr. Alberto Rodríguez Varón & $\begin{array}{l}\text { Abril de } 1998 \text { a diciembre } \\
\text { de } 1999\end{array}$ \\
\hline Dr. Fernando Sierra Arango & $\begin{array}{l}\text { Enero de } 2000 \text { a septiembre } \\
\text { de } 2001\end{array}$ \\
\hline Dr. Luis Alejandro Orozco Linares & $\begin{array}{l}\text { Octubre de } 2001 \text { a } \\
\text { septiembre de } 2002\end{array}$ \\
\hline Dr. Mario Humberto Rey Tovar & $\begin{array}{l}\text { Octubre de } 2002 \text { a } \\
\text { diciembre de } 2003 \text {, octubre } \\
\text { de } 2005 \text { a septiembre de } \\
2007\end{array}$ \\
\hline Dr. Oscar Alfredo Beltrán Galvis & $\begin{array}{l}\text { Enero de } 2004 \text { a septiembre } \\
\text { de } 2005\end{array}$ \\
\hline Dr. Luis Fernando Pineda Ovalle & $\begin{array}{l}\text { Octubre de } 2007 \text { a } \\
\text { diciembre de } 2009\end{array}$ \\
\hline Dr. Rómulo Darío Vargas Rubio & $\begin{array}{l}\text { Enero de } 2010 \text { a diciembre } \\
\text { de } 2011\end{array}$ \\
\hline Dr. John Ospina Nieto & $\begin{array}{l}\text { Enero de } 2012 \text { a marzo de } \\
2012\end{array}$ \\
\hline Dr. Jaime Alvarado Bestene & $\begin{array}{l}\text { Abril de } 2012 \text { a diciembre } \\
\text { de } 2019\end{array}$ \\
\hline Dr. Diego Mauricio Aponte Martín & Desde enero de 2020 \\
\hline
\end{tabular}

Como miembro del comité editorial, considero que se han logrado metas a corto plazo como el mejoramiento en el diseño de la revista, el cumplimiento estricto de las normas de publicación, la inclusión del open research and contributor ID (ORCID) de los autores y del digital object identifier (DOI) de cada artículo, el mejoramiento del portal de la revista y la supresión de la versión impresa.

$\mathrm{El}$ ingreso de la revista a la era digital representa relativas desventajas para algunos, como privarse del agradable olor del papel y la tinta, y la necesidad de depender de la energía eléctrica o de la carga del celular. Pero es innegable que son muchos más los beneficios como la disponibilidad 
inmediata de la información, la facilidad en la difusión de la revista, el incremento de artículos publicados por número y de lectores de los mismos, y la inclusión de videos y enlaces que facilitan aún más el aprendizaje, todo esto favorecido por la ayuda y el impulso dado por las redes sociales; además de que hay una importante reducción de costos y es un modo de difusión más amigable con el medio ambiente.

Estos logros han sido posibles gracias al apoyo de la Asociación Colombiana de Gastroenterología y al acompañamiento y trabajo mancomunado de los representantes de las Asociaciones de Endoscopia Digestiva, Coloproctología y Hepatología.

A mediano plazo continuará el proceso de internacionalización de la revista, debido a que cada vez son más los artículos de autores extranjeros que se publican. Por supuesto, la mayor cantidad de lectores se encuentran en Colombia, pero hay un importante número de lectores de países como México, Perú, Ecuador, Argentina, España e incluso de los Estados Unidos, gracias a la adecuada difusión de la versión digital y al uso de las redes sociales.

Como hay que hacerlo frecuentemente, se ha hecho un alto en el camino, y mirar atrás ha permitido entender la importancia del trabajo desarrollado por todos aquellos que han sido artífices de lo que ahora es la revista y ofrecerles el reconocimiento que se merecen. Se tiene puesta la mirada en el futuro, con una actitud de entrega y trabajo en la búsqueda de caminos y metas claras y comunes para todos, como llegar a Pubmed central, una labor difícil, en la que probablemente habrá tropiezos y caídas, pero que se logrará con el apoyo de todos.

El pasado mes de abril se realizó un seminario web dirigido especialmente a los autores y lectores, en el que se mostró de forma trasparente el actual proceso editorial y el enfoque de la revista, y se explicó nuevamente de una forma muy didáctica los pasos para el envío de un artículo. Además, se reconoció la labor de los editores que han forjado la historia de la revista, el trabajo de la asistente editorial, Sra. Olga Mejía, durante 18 años, de los revisores pares y de los autores que la siguen forjando (Figura 2). En dicho evento, el editor-director mencionó que: "es muy importante conocer y hacer parte de todo el proceso editorial, ser un buen lector, un gran autor, un disciplinado revisor y un responsable editor", todo esto para seguir haciendo de esta "una revista de todos, para todos".



Figura 2. Reconocimiento a autores, revisores pares, asistente editorial y editores.

\section{REFERENCIAS}

1. Mendoza S, Paravic T. Origen, clasificación y desafíos de las Revistas Científicas. Investigación y Postgrado. 2006;21(1):49-75.

2. Reyes H. Historia, propósitos y características de las revistas médicas. Rev Med Chile. 2018;146(8):913-20.
3. Archila PE. 30 años de la Revista Colombiana de Gastroenterología. Rev Col Gastroenterol. 2017;31(4):327-330. 